Vietnam Journal of Mechanics, VAST, Vol. 27, No. 1 (2005), pp. 51-58

\title{
COMPARISON OF VELOCITY DISTRBUTION IN TURBULENT DIFFUSION JET GIVEN BY THE INTEGRAL MODEL AND CODE CFD FLUENT 6.0
}

\author{
Bui Van GA ${ }^{1}$, Pham Thi Kim Loan ${ }^{1}$, Nhan Hong Quang ${ }^{2}$ \\ ${ }^{1}$ Danang University \\ ${ }^{2}$ National Institute of Labour Protection
}

\begin{abstract}
Integral model is simple in utilization, low in CPU time calculation, suitable for a lot of practical applications of turbulent diffusion jet. However the assessment of accuracy of the model should be carried out by experimental data and by results of available multidirectional codes. The present paper shows the comparison of velocity profiles given by the integral model and the CFD FLUENT 6.0 Code. The difference in results given by the two models is lower than $10 \%$ when Reynolds number at the exit nozzle below 5000 .
\end{abstract}

\section{INTRODUCTION}

Diffusion turbulent jet has many engineering applications. Studies of turbulent jet formerly were carried out mainly by basing on the experimental measurements. And the essential rules drawn from these studies have had great practical applications to industry, especially in the field of internal combustion engine. In recent years, making use of the rapid development of information technologies, problems in diffusion turbulent jet have been resolved comprehensively by lots of Computational Fluid Dynamics (CFD) codes. The jet evolution in many different conditions has been fully understood even in the special cases that empirical measurements had many difficulties or were unable to be carried out in the past. However, such codes are often very complicated, demanding intensive calculating expenses and sometimes not suitable to the practical applications.

The urgent need to make out simple solver effectively supporting studies of diffusion turbulent jet has led to preliminary called integral model. This model requires to be established based on the system of integral equations that describe the variation of physical scalars along the jet axis, together with the experimental rules of their fluctuation in the radical direction $[1],[2],[3]$.

An initial integral model has been established to describe the vertical diffusion turbulent jet in still air. This model bears a great significance in verifying the assumptive boundary conditions and the accuracy of experimental results. However in fact, both in the combustion chamber of engine and in the ambient air, diffusion turbulent jets always are affected strongly by the moving of surrounding air. For this reason, a general model has been established to describe the diffusion turbulent jet under the effect of non-stationary surrounding air [4].

Although the numerical results given by integral model showed rather appropriate to experiment in many specific cases [5], [6], on purpose of generalizing for different applications, the integral model requires being carefully validated by the calculating results given by multi-dimensional models. In this paper we present the compared results coming from the integral model and from the code CFD FLUENT 6.0. 
To initially valuate the calculated results, we applied to the vertical jet injecting into still air. Turbulent jets exit from the nozzles with the diameters of 2 and $3 \mathrm{~mm}$. Exit velocity changes from $50 \mathrm{~m} / \mathrm{s}$ to $100 \mathrm{~m} / \mathrm{s}$. Fuel used is Liquefied Petroleum Gas (LPG).

\section{THE GOVERNED EQUATIONS}
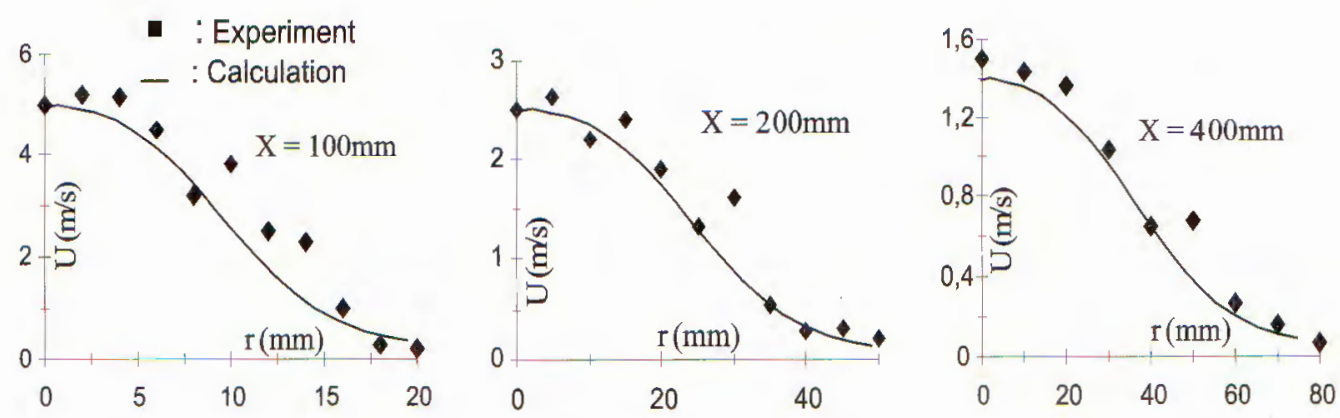

Fig. 1. Comparison of velocity profiles given from integral model and experimental data

The system of equations governing the inclined diffusion turbulent jet in moving air was described in [2]. It consists of the conserved equations written in the integral form and the standard $k$ - $\varepsilon$ model. From the model, the variations of physical quantities along jet axis are defined. And their variations in the radical direction are calculated by using the assumption of "top hat profile" [6].

The integral model has been validated by the measured results of diffusion turbulent jet and flame made in the air ambient and in the combustion chamber of Diesel engines [2]. The comparison of numerical results and experimental data in some representative cases is shown in Fig. 1. Experimental results of the distribution of velocity in diffusion jet were measured by using Laser Doppler Anemometry (LDA) method [3]. In these figures we can see that near the jet axis, the numerical values seem to be rather lower than that of measurement. Fig. 2 shows the representative numerical results of the velocity distribution in diffusion turbulent jet calculated by the integral model.

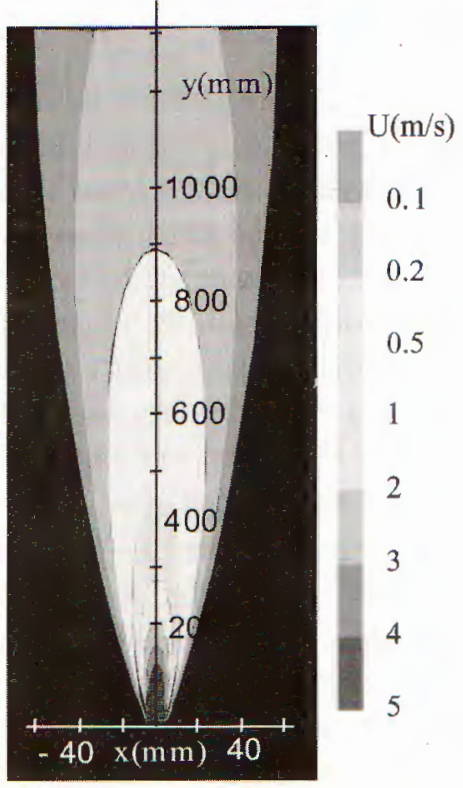

Fig. 2. Velocity distribution of jet calculating from integral model

The system of equations described diffusion jet in CFD code FLUENT is generally written as follows [10].

The continuity equation:

$$
\frac{\partial \bar{\rho}}{\partial t}+\frac{\partial \overline{\rho u_{i}}}{\partial x_{i}}=\frac{\partial \bar{\rho}}{\partial t}+\frac{\partial \bar{\rho} \tilde{u}_{i}}{\partial x_{i}}=0 .
$$

The momentum equations: 


$$
\frac{\partial \bar{\rho} \tilde{u}_{i}}{\partial t}+\frac{\partial \bar{\rho} \tilde{u}_{i} \tilde{u}_{j}}{\partial x_{j}}=-\frac{\partial \bar{p}}{\partial x_{i}}+\frac{\partial}{\partial x_{j}}\left[\mu\left(\frac{\partial \tilde{u}_{i}}{\partial x_{j}}+\frac{\partial \tilde{u}_{j}}{\partial x_{i}}-\frac{2}{3} \delta_{i j} \frac{\partial \tilde{u}_{k}}{\partial x_{k}}\right)\right]-\frac{\partial}{\partial x_{j}}\left(\overline{\rho u_{i}^{\prime \prime} u_{j}^{\prime \prime}}\right) .
$$

In equations $(2.1)$ and $(2.2)$ the tilde $(\sim)$ denotes the Favre average and the bar $(-)$ denotes the Reynolds average; $\mathrm{u}_{i}, \mathrm{u}_{j}=\{\mathrm{u}, \mathrm{v}, \mathrm{w}\}$ and $u_{i}^{\prime \prime} ; u_{j}^{\prime \prime}=\left\{u^{\prime \prime}, v^{\prime \prime}, w^{\prime \prime}\right\}$ are velocity vectors and the fluctuating velocity components respectively; $x_{i}, x_{j}=\{x, y, z\}$ are Cartesian coordinate vectors; $\delta_{i j}$ is the tensorial Kronecker delta $\left(\delta_{i j}=1\right.$ for $i=j$ and $\delta_{i j}=0$ for $i \neq j) ; \mu$ is viscosity; $\rho$ is density and $t$ is time.

The Reynolds stress tensor is modeled by using Boussinesq hypothesis relating the Reynolds stresses to the mean velocity gradients [7]:

$$
-\rho \overline{u_{i}^{\prime} u_{j}^{\prime}}=\mu_{t}\left(\frac{\partial u_{i}}{\partial x_{j}}+\frac{\partial u_{j}}{\partial x_{i}}\right)-\frac{2}{3}\left(\rho k+\mu_{t} \frac{\partial u_{i}}{\partial x_{i}}\right) \delta_{i j} .
$$

Here $\mu_{\tau}$ is eddy viscosity. The turbulence kinetic energy, $k$, and its rate of dissipation, $\varepsilon$, are obtained from the following transport equations of the standard $k-\varepsilon$ model as following (Launder and Spalding [8]):

$$
\begin{aligned}
& \frac{\partial}{\partial t}(\rho k)+\frac{\partial}{\partial x_{i}}\left(\rho k \bar{u}_{i}\right)=\frac{\partial}{\partial x_{j}}\left(\left(\mu+\frac{\mu_{\tau}}{\sigma_{k}}\right) \frac{\partial k}{\partial x_{j}}\right)+G_{k}+G_{b}-\rho \varepsilon-Y_{M}+S_{k}, \\
& \frac{\partial}{\partial t}(\rho \epsilon)+\frac{\partial}{\partial x_{i}}\left(\rho \epsilon \bar{u}_{i}\right)=\frac{\partial}{\partial x_{j}}\left(\left(\mu+\frac{\mu_{\tau}}{\sigma_{k}}\right) \frac{\partial k}{\partial x_{j}}\right)+C_{l \varepsilon} \frac{\varepsilon}{k}\left(G_{k}+C_{3 \varepsilon} G_{b}\right)-C_{2 \varepsilon} \rho \frac{\varepsilon^{2}}{k}+S_{\varepsilon}
\end{aligned}
$$

$k$ and $\varepsilon$ are defined:

$$
k=\frac{1}{2} \overline{u_{i}^{\prime} u_{i}^{\prime}}, \quad \varepsilon=\nu \overline{\frac{\partial u_{i}^{\prime}}{\partial x_{j}} \frac{\partial u_{i}^{\prime}}{\partial x_{j}}} .
$$

In these equations:

- $\sigma_{k}$ and $\sigma_{\varepsilon}$ are the turbulent Prandtl numbers for $\mathrm{k}$ and $\varepsilon$ respectively $\left(\sigma_{k}=1.0, \sigma_{\varepsilon}\right.$ $=1.3)$. $S_{k}$ and $S_{\varepsilon}$ are the user-defined source terms.

- $G_{k}$, representing the production of turbulence kinetic energy, is modeled identically for the standard $k-\varepsilon$ model. From the exact equation for the transport of $k$, this term may be defined as (in a manner consistent with the Boussinesq hypothesis):

$$
G_{k}=\mu_{\tau} S^{2}
$$

where $S=\sqrt{2 S_{i j} S_{i j}}$ is swirl number and $S_{i j}$ is mean strain-rate tensor.

- $G_{b}$ is the generation of turbulence due to buoyancy:

$$
G_{b}=\beta g_{i} \frac{\mu_{t}}{P r_{t}} \frac{\partial T}{\partial x_{i}}
$$

- $P r_{t}$ is the turbulent Prandtl number for energy, and for the standard $k-\varepsilon$ model, the default value of $P r_{t}$ is $0.85 ; g_{i}$ is the component of the gravitational vector in the $i$-th direction. $T$ is temperature. The coefficient of thermal expansion $\beta$ is defined as:

$$
\beta=-\frac{1}{\rho}\left(\frac{\partial \rho}{\partial T}\right)_{p}
$$


where the symbol ()$_{p}$ denotes the isobaric condition.

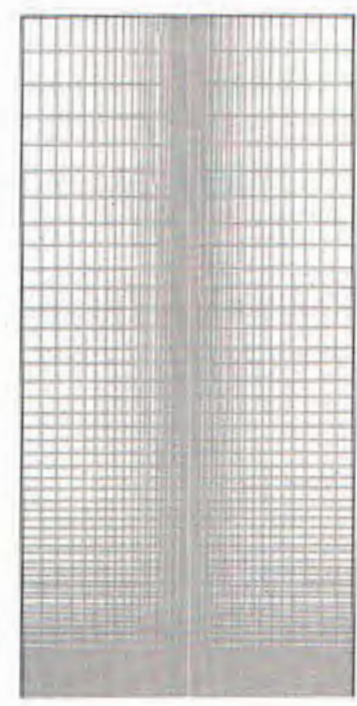

a.

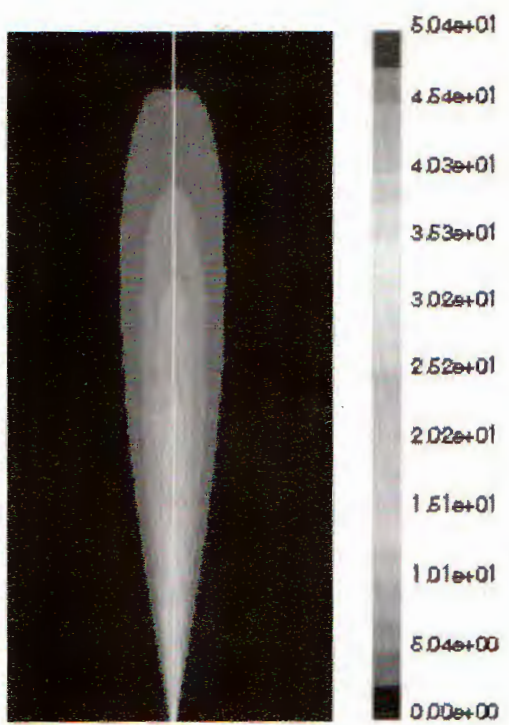

b.

Fig. 3. Calculation of vertical jet 2D

a. Making grid

b. Representative calculated result

While the buoyancy effects on the generation of $k$ are relatively well understood, the effect on $\varepsilon$ is less clear. In FLUENT, by default, the buoyancy effects on $\varepsilon$ are neglected simply by setting $G_{b}$ to zero in the transport equation for $\varepsilon$. However, the degree to which $\varepsilon$ is affected by the buoyancy is determined by the constant $C_{3 \varepsilon}$. In FLUENT, $C_{3 \varepsilon}$ is not specified, but is instead calculated according to the following relation [9]:

$$
C_{3 \varepsilon}=\tanh \left|\frac{v}{u}\right|
$$

where $v$ and $u$ are the components of the flow velocity, which is parallel and perpendicular to the gravitational vector. In this way, $C_{3 \varepsilon}$ will be 1 when the main flow direction is aligned with the direction of gravity. For velocity direction that are perpendicular to the gravitational vector $C_{3 \varepsilon}$ will be zero [8].

- $Y_{M}$ represents the volumetric change in the flows which is normally neglected in the modeling of incompressible flows. In the compressible flows, this term is modeled according to a proposal by Sarkar [7]:

$$
Y_{M}=2 \rho \varepsilon M_{t}^{2}
$$

where $M_{t}$ is the turbulent Mach number, defined as:

$$
M_{t}=\sqrt{\frac{k}{a^{2}}}
$$


and $a$ is the speed of sound in gas.

- As in other $k-\varepsilon$ models, the eddy viscosity $\mu_{\tau}$ is computed from:

$$
\mu_{t}=\rho C_{\mu} \frac{k^{2}}{\varepsilon}
$$

- $C_{1 \varepsilon}, C_{2 \varepsilon}, C_{3 \varepsilon}$ and $C_{\mu}$ are constants. In FLUENT the model constants are: $C_{1 \varepsilon}=$ $1.44, C_{2 \varepsilon}=1.92, C_{\mu}=0.09$.

The equations are then solved numerically. The equations have to be discretized. The discretization is achieved by approximating the governing equations with algebraic expressions. Conserved equations are applied to each control volume and the values of variables are calculated at the center of the volume [11]. Interpolation is then used to get values at the surface of the control volume and there are several ways to do this. The Upwind Interpolation Scheme is used here in the study [10].

\section{MAKING MESH FOR JET}

Meshes were made with the help of the software Gambit 2.1. In case of vertical turbulent jet, axi-symmetric domain is chosen with the diameter of 500 and the height of $1000 \mathrm{~mm}$. Grid steps was made small in the jet axis. The farer from the jet axis was, the larger steps were made. And the same way was done in the axial direction. Mesh contains 2600 quadrilaterál cells and 2727 nodes. Calculating mesh is shown in Fig. 3a.

Boundary condition at the inlet of calculating domain was defined as velocity-inlets changing from $50 \mathrm{~m} / \mathrm{s}$ to $100 \mathrm{~m} / \mathrm{s}$ with a turbulence intensity of $10 \%$ and hydraulic diameters of $0.002 \mathrm{~m}$ and $0.003 \mathrm{~m}$. The domain sides are all defined as axis-symmetric. Pressure-outlets boundary is set at the top of calculated domain.

Fig. 3b shows the representative calculating result in the case of vertical diffusion turbulent jet in still air.

\section{RESULTS AND DISCUSS}

The velocity distributions in the cross-sections given by the integral model and FUENT 6.0 are shown in the Fig. 4. The same set of constants is chosen for calculation in the two models. While velocity profiles in multi-dimension model are built by resolving the conserved equations for each point of calculating domain in the radical direction, for integral model they are determined conforming to the similar rule profnsed by Ebrahini [9]:

$$
U(\eta)=U_{c} \exp \left(-\eta^{2} \ln (2)\right)
$$

where $U_{c}$ is the maximum velocity in jet axis, $\eta$ is a dimensionless length:

$$
\eta=\frac{r}{r_{0.5}}
$$

where $r$ is radial coordinate and $r_{0,5}$ is the jet diameter at which the velocity is equal to $0.5 U_{c}$.

With the low exit velocities, the Reynolds number at exit nozzle is about 3000 , difference between the two models is very small, mainly occurring in the vicinity of jet axis 
(Fig.4). Velocity profiles of the two models nearly coincide. This means the similar rule (4.1) is conformity with turbulent diffusion jet.
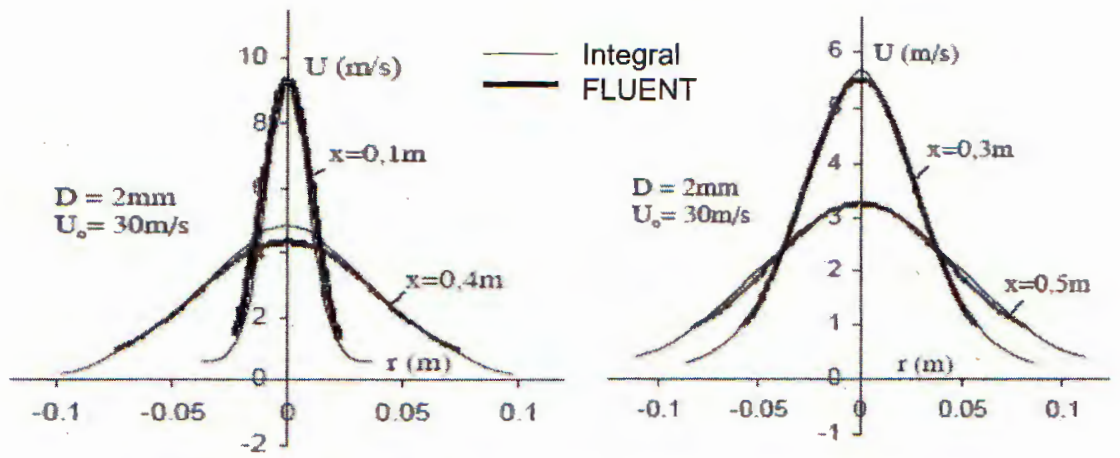

Fig.4. Comparison velocity profiles given by integral model and FLUENT

$\left(\mathrm{D}=2 \mathrm{~mm}, \mathrm{U}_{0}=30 \mathrm{~m} / \mathrm{s}, \mathrm{Re}=3.000\right)$
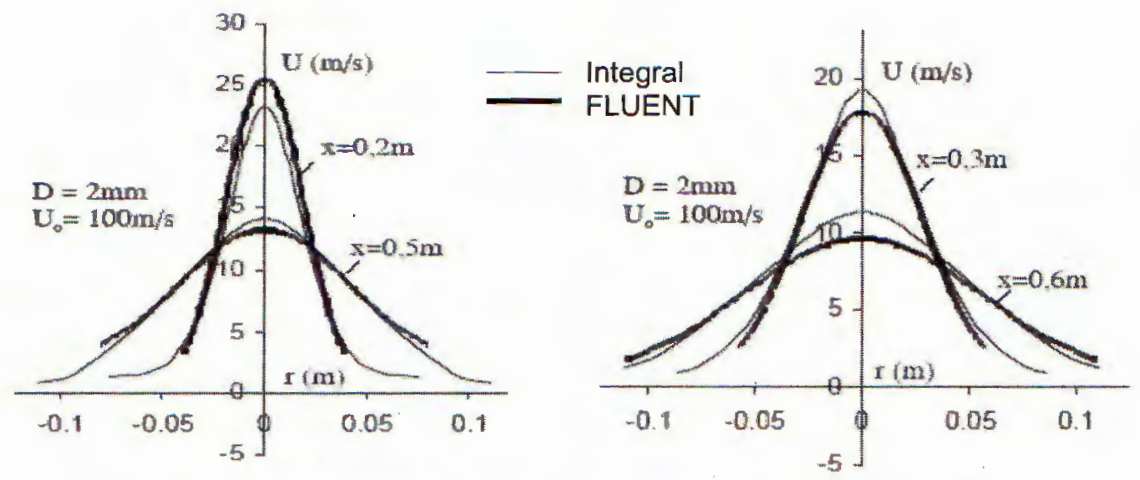

Fig.5. Comparison velocity profiles given by integral model and FLUENT $\left(D=2 \mathrm{~mm}, U_{0}=100 \mathrm{~m} / \mathrm{s}, R e=10.000\right)$
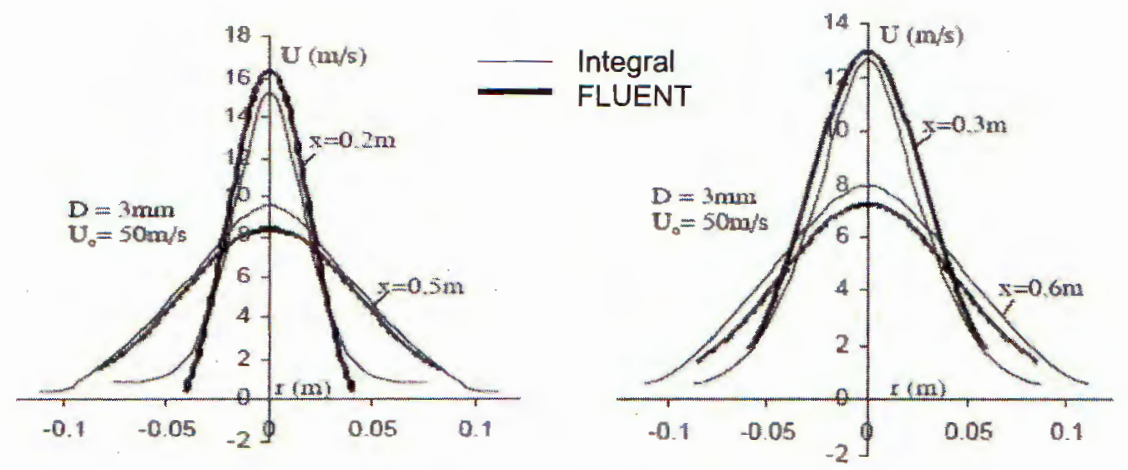

Fig.6: Comparison velocity profiles given by integral model and FLUENT $\left(D=3 \mathrm{~mm}, U_{0}=50 \mathrm{~m} / \mathrm{s}, R e=7.500\right)$ 
When the Reynolds number at the exit nozzle increases, the difference in velocity profiles given by the two models increases simultaneously (Fig. 5). With the exit nozzle diameter of $2 \mathrm{~mm}$ and the exit velocity of $100 \mathrm{~m} / \mathrm{s}$ (Reynolds number of 10.000), the velocity difference reaches the maximum value of about $15 \%$. The same difference is obtained when exit nozzle diameter increases to $3 \mathrm{~mm}$ with the Reynolds number of 7.500 (Fig. 6).

Application of "top hat profile" assumption to the integral model caused difference in velocity profiles in the vicinity of jet axis. According to the hypothesis, velocity in each cross-section of jet is purposed to be constant so that the integration of mass flow and momentum in the section is conserved.

From the above comparison we can see that calculated results given from the integral model good coincide with that given from the FLUENT code in the case of turbulent jets with low Reynolds number. If considering the maximum difference of velocity between the two models as a function of the Reynolds number of flows at exit nozzle, the above study shows that for flows with the Reynolds number below 5000, the difference is within 10\%. This limit of Reynolds number is suitable with nearly all injection systems applied to field of internal combustion engine.

\section{CONCLUSSION}

The integral model with assumption of "top hat profile" in the radical direction gives the numerical results suitable with that of the multi-dimensional model for flows having Reynolds number at the exit nozzle below 5000. In this meaning, the integral model is capable of application to almost jet configurations in inner combustion engine with a maximum error below $10 \%$ in comparison with the multi-dimensional.

The velocity profiles given by the two models almost agree with each other. Application of "top hat profile" assumption to the integral model for integrating mass flow and momentum caused the velocity values at the jet axis smaller than that given by multidimensional model.

Acknowledgments. This work has been fulfilled with the sponsor of the National Fundamental Research Program, Project Number 320604.

\section{REFERENCES}

1. Bui Van Ga, An Integral Model for Calculation of LPG Jet Development in Combustion Chamber of Spark Ignition Engine, International Conference on HPSC, Hanoi 10-14 March 2003.

2. Bui Van Ga, Pham Xuan Mai, Liviu GEORGESCU: A mathematical model for calculation of turbulence diffusion combustion in air and in Diesel engines, Proceedings of the VII International Conference of Motor Vehicles CAR-2000 (FISITA, SIAR), Romania, ICE (2000) 8-16.

3. Bui Van Ga, Phung Xuan Tho, Nhan Hong Quang, Nguyen Huu Huong, Phenomenological model for determining velocity field of LPG jet in combustion chamber of direct injection SI engine, Vietnam Journal of Mechanics 26 (2004) 83-92.

4. Bui Van Ga, Nhan Hong Quang, J. M. Vignon, Calculation of turbulent diffusion jets under effects of gravity and moving surrounding air, Vietnam Journal of Mechanics 23 (2001) 87-94. 
5. Bui Van Ga, Duong Viet Dung, Tran Van Nam, Simulation of liquefied petroleum gas jet in combustion chamber of spark ignition engine, Journal of Science and Technology 39 (30-31) (2001) 97-103.

6. Bui Van Ga, Duong Viet Dung, Tran Van Nam, Simulation of liquefied petroleum gas jet in combustion chamber of spark ignition engin, Vietnam Journal of Mechanics 24 (2002) 209-218.

7. S. Sarkar, L. Balakrishnan, Application of a Reynolds-Stress Turbulence Model to the Compressible Shear Layer, ICASE Report 90-18, NASA CR 182002, 1990.

8. B. E. Launder, D. B. Spalding, Lectures in Mathematical Models of Turbulence, Academic Press, London, England, 1972.

9. I. Ebrahini, R. Kleine, The nozzle fluid concentration fluctuation field in round turbulent fuel jets and jet diffusion flames, Sixteenth Symposium (International) on Combustion, 1976, pp. 1711-1723.

10. Fluent 6.0 User's Guide, Fluent. Inc., Cennterra Resource Park, Lebanon, NH 30766, 2002.

11. J. Y. Murthy, S. R. Mathur, A Finite Volume Method for Radiative Heat Transfer Using Unstructured Meshes, AIAA-98-0860, 1998.

Received December 1, 2004

Revised May 20, 2005

\section{SO SÁNH TRƯờng TỐC Độ CƯA TIA PHUN KHUẾCH TÁN RỐI TÍNH THEO MÔ HİNH TÍCH PHÂN VÀ CHƯƠNG TRİNH FLUENT 6.0}

Mô hình tích phân một chiều đơn giản, cho kết quả nhanh chóng, phù hợp với nhiều áp dụng thực tiễn đối với tia phun khuếch tán rối. Tuy nhiên độ chính xác của mô hình cần được đánh giá, bằng số liệu thực nghiệm và bằng cách so sánh với kết quả cho bởi các phần mềm của mô hình nhiều chiều có sẵn. Bài báo này so sánh trường tốc độ cho bởi mô hình tích phân và chương trình Fluent 6.0. Sai lệch giữa hai mô hình nằm trong giới hạn 1\% khi số Reynolds ở miệng vòi phun dưới 5000. 